\title{
Evaluating CT Perfusion Using Outcome Measures of Delayed Cerebral Ischemia in Aneurysmal Subarachnoid Hemorrhage
}

\author{
P.C. Sanelli, N. Anumula, C.E. Johnson, J.P. Comunale, A.J. Tsiouris, H. Riina, A.Z. Segal, P.E. Stieg, R.D. Zimmerman, and A.I. Mushlin
}

因䟚

\begin{abstract}
BACKGROUND AND PURPOSE: DCI is a serious complication following aneurysmal SAH and remains a leading cause of morbidity and mortality. Our aim was to evaluate CTP in aneurysmal SAH by using outcome measures of DCI.

MATERIALS AND METHODS: This was a retrospective study of consecutive patients with SAH enrolled in a prospective institutional review board-approved clinical accuracy trial. Qualitative CTP deficits were determined by 2 neuroradiologists blinded to clinical and imaging data. Quantitative CTP was performed by using a standardized protocol with region-of-interest placement sampling of the cortex. Primary outcome measures were permanent neurologic deficits and infarction. The secondary outcome measure was DCl, defined as clinical deterioration. CTP test characteristics $(95 \% \mathrm{Cl})$ were determined for each outcome measure. Statistical significance was calculated by using the Fisher exact and Student $t$ tests. ROC curves were generated to determine accuracy and threshold analysis.

RESULTS: Ninety-six patients were included. Permanent neurologic deficits developed in 33\% (32/96). CTP deficits were seen in 78\% $(25 / 32)$ of those who developed permanent neurologic deficits and $34 \%(22 / 64)$ of those without $(P<.0001)$. CTP deficits had $78 \%$ $(61 \%-89 \%)$ sensitivity, $66 \%(53 \%-76 \%)$ specificity, and $53 \%(39 \%-67 \%)$ positive and $86 \%(73 \%-93 \%)$ negative predictive values. Infarction occurred in $18 \%$ (17/96). CTP deficits were seen in $88 \%(15 / 17)$ of those who developed infarction and $41 \%(32 / 79)$ of those without $(P=$ .0004). CTP deficits had an $88 \%(66 \%-97 \%)$ sensitivity, 59\% (48\%-70\%) specificity, and $32 \%(20 \%-46 \%)$ positive and $96 \%(86 \%-99 \%)$ negative predictive values. DCI was diagnosed in 50\% (48/96). CTP deficits were seen in $81 \%(39 / 48)$ of patients with DCl and in $17 \%(8 / 48)$ of those without $(P<.0001)$. CTP deficits had $81 \%$ (68\%-90\%) sensitivity, $83 \%(70 \%-91 \%)$ specificity, and $83 \%(70 \%-91 \%)$ positive and $82 \%$ (69\%-90\%) negative predictive values. Quantitative CTP revealed significantly reduced CBF and prolonged MTT for DCI, permanent neurologic deficits, and infarction. ROC analysis showed that CBF and MTT had the highest accuracy.
\end{abstract}

CONCLUSIONS: CTP may add prognostic information regarding DCl and poor outcomes in aneurysmal SAH.

ABBREVIATIONS: $\mathrm{AUC}=$ area under the curve; $\mathrm{Cl}=$ confidence interval; $\mathrm{DCl}=$ delayed cerebral ischemia; $\mathrm{ROC}=$ receiver operating characteristic analysis; $\mathrm{SE}=$ standard error

D $\mathrm{CI}$ is a serious complication following aneurysmal SAH and remains the leading cause of morbidity and mortality. Symptoms typically develop between 4 and 14 days after aneurysm rupture. Vasospasm is considered a contributing factor to the devel-

Received January 9, 2012; accepted after revision April 20.

From the Departments of Radiology (P.C.S., N.A., C.E.J., J.P.C., A.J.T., R.D.Z.), Neurological Surgery (H.R., P.E.S.), Neurology (A.Z.S.), and Public Health (P.C.S., A.I.M.), Weill Cornell Medical College, New York-Presbyterian Hospital, New York, New York.

This work was supported by grant 5K23NS058387-02 from the National Institute of Neurological Disorders and Stroke, a component of the National Institutes of Health.

The contents of this article are solely the responsibility of the authors and do not necessarily represent the official view of NINDS or NIH.

Please address correspondence to Pina C. Sanelli, MD MPH, Department of Radiology, Weill Cornell Medical College, New York-Presbyterian Hospital, 525 East 68th St, Starr 630, New York, NY 10065; e-mail: pcs9001@med.cornell.edu opment of DCI, in which severe arterial narrowing may result in decreased cerebral blood flow. Early diagnosis and prompt treatment of DCI are focused primarily on preventing its devastating sequelae of permanent neurologic deficits, cerebral infarction, and death.

Currently, there are several methods used in clinical practice to support a diagnosis of DCI, including clinical examination, neurologic monitoring devices, transcranial Doppler sonography, CTA, CTP, MR imaging with diffusion and perfusion imaging, and DSA. Often, the clinical and imaging findings do not correlate; this difference may lead to delayed diagnosis and inconsistent terminology used to describe DCI. As a result, it has be-

\footnotetext{
- Indicates open access to non-subscribers at www.ajnr.org

Indicates article with supplemental on-line tables.

http://dx.doi.org/10.3174/ajnr.A3225
} 
come challenging to understand the true impact of imaging and interventions in this patient population. Recent expert consensus has recommended a uniform definition of DCI and its outcomes for use in clinical research. ${ }^{1}$ The primary outcome measures of DCI are functional disability and cerebral infarction not attributed to other causes. The diagnosis of DCI, defined by clinical deterioration, is recommended as a secondary outcome measure because it is suspected of having lower interobserver agreement rates. ${ }^{1}$ Together, these outcome measures capture the most relevant elements of cerebral ischemia after SAH and therefore are valuable for assessing imaging and treatment strategies for DCI. Furthermore, the term "vasospasm" is reserved for arterial narrowing on imaging studies and is not recommended for use as an outcome measure itself because almost half of patients with severe vasospasm do not have DCI. ${ }^{2}$

Several studies support the use of perfusion imaging for detection of hemodynamic disturbances thought to occur in DCI and vasospasm. $^{3-7}$ There are several advantages in using CTP in this critically ill population, including its noninvasiveness, short acquisition time, widespread availability, and limited patient contraindications. Additionally, CTP deficits have been shown to have high sensitivity and specificity for detection of DCI and vasospasm. ${ }^{3-7}$ However, to our knowledge, few studies have evaluated CTP by using outcome measures of DCI to fully understand its impact in this patient population. CTP has the potential to add important prognostic information regarding poor outcomes for guiding management and treatment decisions.

The purpose of this study was to evaluate qualitative and quantitative CTP by using the primary and secondary outcome measures of DCI in aneurysmal SAH. The primary outcome measures were permanent neurologic deficits and infarction. The secondary outcome measure was DCI, defined as clinical deterioration. Our hypothesis was that there are significant differences in the CTP parameters in patients with DCI and poor outcomes; therefore, CTP may have the potential to provide valuable prognostic information.

\section{MATERIALS AND METHODS Study Design}

A retrospective cohort study of 104 consecutive patients with aneurysmal SAH enrolled in a prospective institutional review board-approved clinical accuracy trial from December 2004 to December 2008 was performed. Inclusion criteria were adult patients (18 years of age and older) with documented aneurysmal SAH at admission based on initial noncontrast head CT, CSF analysis, CTA, and/or DSA. Exclusion criteria were CTP performed after treatment of DCI or after cerebral infarction occurred. All subjects in the study underwent surgical clipping and/or endovascular coiling for aneurysm repair and were monitored in the neurologic intensive care unit, as per the usual standard of care. Chart review was performed for the clinical and demographic characteristics of the study population. Close clinical observation assessed clinical deterioration not explained by other causes such as aneurysm rebleeding, intracranial hemorrhage, hydrocephalus, infection, metabolic disturbance, and seizure. Patients were evaluated for treatment on the basis of clinical deterioration, neurologic monitoring, transcranial Doppler sonography, and DSA. Discharge status was classified as home, rehabilitation care facility (acute rehabilitation or long-term skilled nursing), and death.

\section{Outcome Measures}

The outcome measures for this study were based on the most relevant clinical and imaging criteria recommended by the recent expert consensus for use in research studies investigating DCI. ${ }^{1}$ The primary outcome measures included the following: 1) cerebral infarction on CT or MR imaging within 6 weeks after SAH, which was not present on imaging up to 48 hours after aneurysm occlusion and was not attributable to other causes such as surgical clipping, endovascular treatment, ventricular catheter placement, and intraparenchymal hematoma ${ }^{1}$ (this definition of cerebral infarction has been used to effectively exclude primary brain damage from SAH and/or surgical interventions), and ${ }^{8,9}$ 2) permanent neurologic deficit on clinical examination, distinct from the deficit at baseline, produced by aneurysm rupture or surgical intervention, and not attributable to other causes.

The secondary outcome measure was DCI, defined as clinical deterioration with the occurrence of focal neurologic impairment (such as hemiparesis, hemiplegia, aphasia, and so forth) or a decrease of at least 2 points on the Glasgow Coma Scale that was not apparent immediately after aneurysm occlusion and was not attributed to other causes by clinical assessment, CT or MR imaging, and laboratory studies.

\section{CTP Protocol, Postprocessing, and Data Collection}

CTP was performed during the typical time period for DCI, between days 6 and 8, in asymptomatic patients and on the same day that clinical deterioration occurred in symptomatic patients. There is a standard scanning protocol for CTP at our institution by using LightSpeed or Pro 16 scanners (GE Healthcare, Milwaukee, Wisconsin) with cine $4 \mathrm{i}$ scanning mode and a 45 -second acquisition at 1 rotation per second with $80 \mathrm{kV}$ (peak) and $190 \mathrm{~mA}$. A scanning volume of $2.0 \mathrm{~cm}$ was used, consisting of 4 sections at 5.0-mm thickness with its inferior extent selected at the level of the basal ganglia, above the orbits, to minimize radiation exposure to the lenses. Approximately $45 \mathrm{~mL}$ of nonionic iodinated contrast was administered intravenously at $5 \mathrm{~mL} / \mathrm{s}$ by using a power injector with a 5-second delay.

Postprocessing of the acquired images into CBF, MTT, and CBV maps was performed on an Advantage Workstation by using CTP software, Version 3.0 (GE Healthcare). This software uses a deconvolution method, which is considered most accurate for low-contrast-injection rates. ${ }^{10}$ The postprocessing technique was standardized for all patients according to recommended guidelines ${ }^{11}$ with the arterial input function as the $\mathrm{A} 2$ segment of the $\mathrm{ACA}^{12}$ and venous function as the superior sagittal sinus.

The perfusion maps were qualitatively evaluated by 2 neuroradiologists (with 7 and 10 years' experience) blinded to clinical and imaging data, to determine the presence of perfusion deficits, de-

fined as areas of decreased CBF and/or elevated MTT. Focal perfusion abnormalities due to the primary hemorrhagic event and surgical intervention, as identified on the acquired images from the CTP dataset, were not included as perfusion deficits related to 
DCI. After the images were reviewed independently, consensus judgment was determined.

Quantitative analysis was conducted by using a standardized method with contiguous region-of-interest placement, measuring $157 \mathrm{~mm}^{2}$, sampling the cerebral cortex. Each CTP section had up to 24 ROIs distributed in the following territories: approximately 6 ROIs in the anterior cerebral artery, 12 in the MCA, and 6 in the posterior cerebral artery. CTP studies were analyzed with readers blinded to all clinical and imaging data to limit test-review bias.

\section{Statistical Analysis}

Only CTP examinations performed before treatment for DCI and before infarction were included in the analysis, to minimize confounding bias because both treatment and infarction may affect the perfusion maps. The incidence of qualitative CTP deficits was calculated for each outcome group. Two-tailed $P$ values were calculated by using the Fisher exact test to determine statistical significance. CTP test characteristics (sensitivity, specificity, and positive and negative predictive values) were determined by using $2 \times 2$ contingency tables, and the $95 \%$ CI was calculated.

Quantitative CTP data were analyzed by calculating the mean $\mathrm{CBF}, \mathrm{CBV}$, and MTT and their SDs for each outcome group. For patients with focal perfusion deficits, ROIs within the affected region were isolated and arithmetic means were calculated. In patients without focal deficits, all ROIs for all 4 section locations were included in the arithmetic means. To minimize the contribution of vascular pixels from large vessels, we excluded CBF values of $>100 \mathrm{~mL} / 100 \mathrm{~g} / \mathrm{min}$ from the statistical analysis and did not use these in calculating the mean CBF, CBV, and MTT, because this method has been published in the evaluation of ischemia. ${ }^{13}$ The ROIs in the perfusion abnormalities due to the primary hemorrhagic event and/or surgical intervention were not included in the statistical analysis. The normality of the distribution of continuous variables was assessed by plotting histograms and the Shapiro-Wilks test. A Student 2-tailed $t$ test was used to determine statistical significance, accepted at $P<.05$. The statistical analysis was performed by using GraphPad software (GraphPad Software, La Jolla, California).

ROC curves were generated for each CTP parameter with its 95\% CI by using the maximum-likelihood fit of a binormal model. The AUC and SE were calculated to quantify accuracy. Diagnostic threshold values were determined by using the northwest corner method, representing the point value on the ROC curve with the greatest discrimination ability between outcome groups. The statistical ROC analysis was performed by using the JLABROC4 1.01 software program (Johns Hopkins University, Baltimore, Maryland).

Using the statistical methods described, we performed a subgroup analysis to evaluate CTP in the DCI (symptomatic) and no DCI (asymptomatic) groups separately, for the primary outcome measures of permanent neurologic deficits and infarction.

\section{RESULTS}

\section{Study Population Characteristics}

A total of 96 patients with aneurysmal SAH were included in the statistical analysis from the 104 patients enrolled in the prospec-
Table 1: Clinical and demographic characteristics of the study population

\begin{tabular}{|c|c|c|c|}
\hline & $\begin{array}{c}\text { All } \\
(n=96)\end{array}$ & $\begin{array}{c}\mathrm{DCl} \\
(n=48)\end{array}$ & $\begin{array}{l}\text { No DCl } \\
(n=48)\end{array}$ \\
\hline Age (yr) (mean) & 50.5 & 52.5 & 48.5 \\
\hline Range & $28-80$ & $29-80$ & $28-78$ \\
\hline Female & $74 \%(71 / 96)$ & $77 \%(37 / 48)$ & $71 \%(34 / 48)$ \\
\hline Male & $26 \%(25 / 96)$ & $23 \%(11 / 48)$ & $29 \%(14 / 48)$ \\
\hline \multicolumn{4}{|l|}{ Aneurysm location } \\
\hline Anterior & $92 \%(88 / 96)$ & $94 \%(45 / 48)$ & $90 \%(43 / 48)$ \\
\hline Posterior & $8 \%(8 / 96)$ & $6 \%(3 / 48)$ & $10 \%(5 / 48)$ \\
\hline \multicolumn{4}{|l|}{ Aneurysm treatment } \\
\hline Surgical clipping & $55 \%(53 / 96)$ & $58 \%(28 / 48)$ & $52 \%(25 / 48)$ \\
\hline Endovascular coiling & $45 \%(43 / 96)$ & $42 \%(20 / 48)$ & $48 \%(23 / 48)$ \\
\hline \multicolumn{4}{|l|}{ Hunt and Hess } \\
\hline Low, grades 1 and 2 & $50 \%(48 / 96)$ & $37 \%(18 / 48)$ & $63 \%(30 / 48)$ \\
\hline High, grades 3,4 , and 5 & $50 \%(48 / 96)$ & $63 \%(30 / 48)$ & $37 \%(18 / 48)$ \\
\hline
\end{tabular}

Table 2: Mean quantitative CTP parameters and SD for the primary outcome measures of permanent neurologic deficits and infarction

\begin{tabular}{|c|c|c|c|c|c|c|c|c|c|c|}
\hline & \multicolumn{2}{|c|}{$\begin{array}{c}\text { Neurologic } \\
\text { Deficits } \\
(n=32) \\
\end{array}$} & \multicolumn{2}{|c|}{$\begin{array}{l}\text { No Deficit } \\
(n=64)\end{array}$} & \multirow{2}{*}{$\begin{array}{c}P \\
\text { Value }\end{array}$} & \multicolumn{2}{|c|}{$\begin{array}{c}\text { Infarction } \\
(n=17)\end{array}$} & \multicolumn{2}{|c|}{$\begin{array}{c}\text { No } \\
\text { Infarction } \\
(n=79) \\
\end{array}$} & \multirow{2}{*}{$\begin{array}{c}P \\
\text { Value }\end{array}$} \\
\hline & Mean & SD & Mean & SD & & Mean & SD & Mean & SD & \\
\hline & 28.68 & 14 & & & & & & 34.61 & & \\
\hline CBV & & 0.7 & & & & & & 91 & 0.54 & \\
\hline MTT & 7.19 & 3.92 & 5.33 & 2.06 & .0029 & 7.8 & 4.2 & 5.55 & 2.45 & 5.0037 \\
\hline
\end{tabular}

tive clinical accuracy trial. Eight patients were excluded for the following reasons: CTP examinations were not performed before treatment for DCI $(n=4)$, CTP acquired data were not retrievable from the archives for postprocessing $(n=3)$, and postprocessing could not be performed due to severe motion degradation $(n=1)$. The clinical and demographic characteristics of the study population are shown in Table 1. Given that Hunt and Hess grades on admission are a clinical predictor of DCI, our study population revealed that almost two-thirds of patients with DCI had high Hunt and Hess grades, whereas almost two-thirds of patients without DCI had low Hunt and Hess grades. Qualitative CTP deficits were seen in 49\% (47/96) of patients. CTP was performed at a median of day 7 after aneurysm rupture.

\section{Primary Clinical Outcome: Permanent Neurologic Deficit}

Permanent neurologic deficits occurred in 33\% (32/96) of patients, and the remaining 67\% (64/96) had no deficit. Qualitative CTP deficits were seen in 78\% (25/32) of patients who developed permanent neurologic deficits and in 34\% (22/64) who did not $(P<.0001)$. The test characteristics $(95 \% \mathrm{CI})$ of qualitative CTP deficits for detecting patients who develop permanent neurologic deficits were $78 \%(61 \%-89 \%)$ sensitivity, 66\% (53\%-76\%) specificity, and 53\% (39\%-67\%) positive and 86\% (73\%-93\%) negative predictive values. Table 2 demonstrates the mean quantitative CTP parameters for these outcome groups. Figure $1 A$ displays the ROC curves for each CTP parameter for detecting patients who develop permanent neurologic deficits. The diagnostic accuracy (SE) for each CTP parameter is $0.66(0.06)$ for CBF, 0.48 (0.06) for CBV, and 0.66 (0.06) for MTT. The CBF threshold of 25 $\mathrm{mL} / 100 \mathrm{~g} / \mathrm{min}$ (75\% sensitivity, 50\% specificity) and an MTT of 4.8 seconds (70\% sensitivity, $50 \%$ specificity) were determined. 


\section{A Neurologic deficits}

CBF

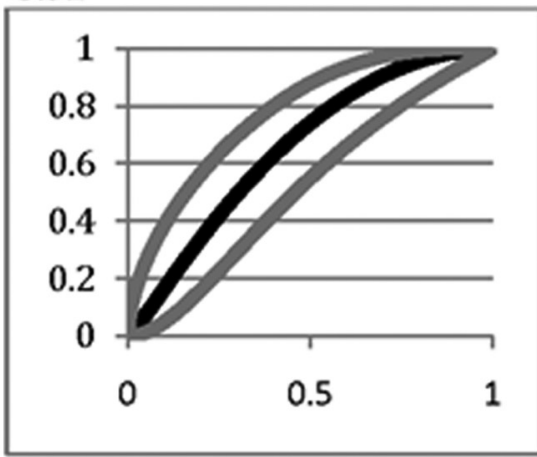

CBV

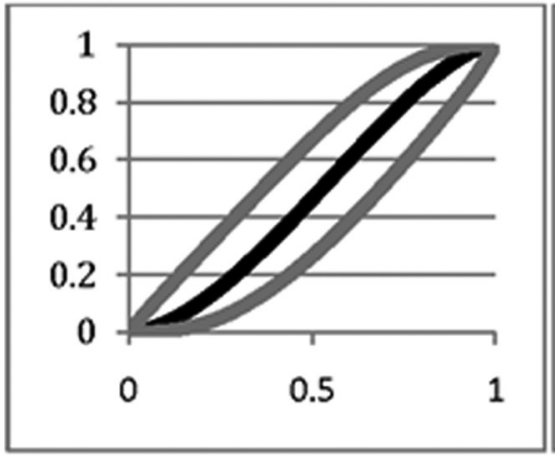

MTT

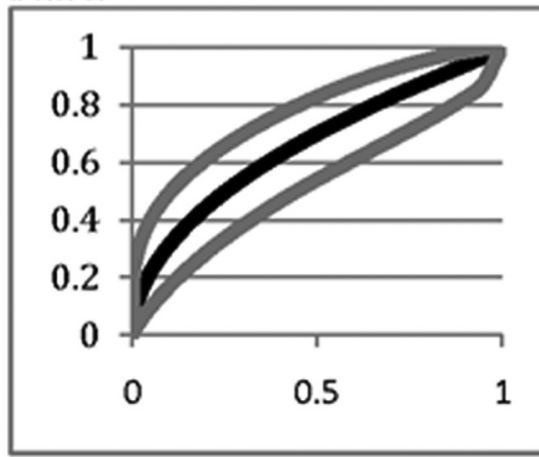

B Cerebral infarction

CBF

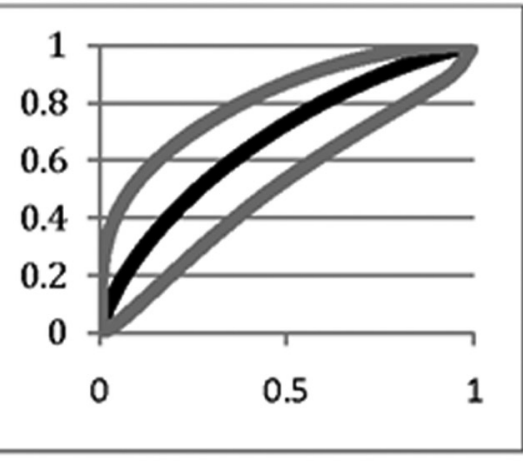

\section{CBV}

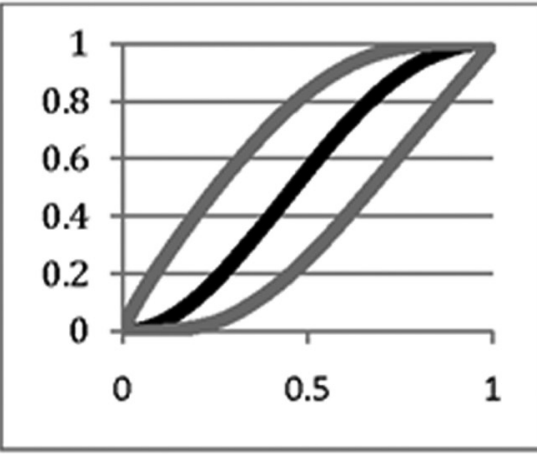

\section{MTT}

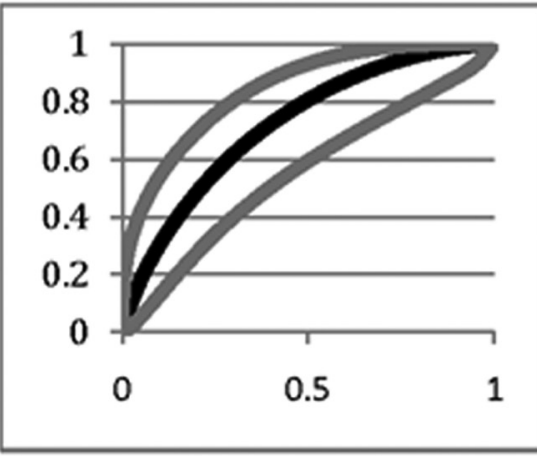

\section{DCI}

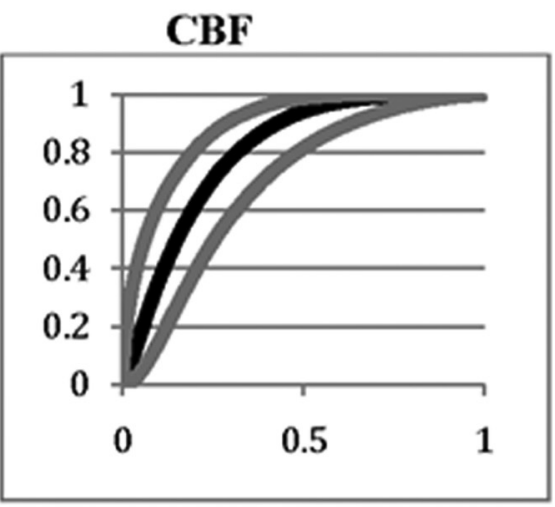

\section{CBV}

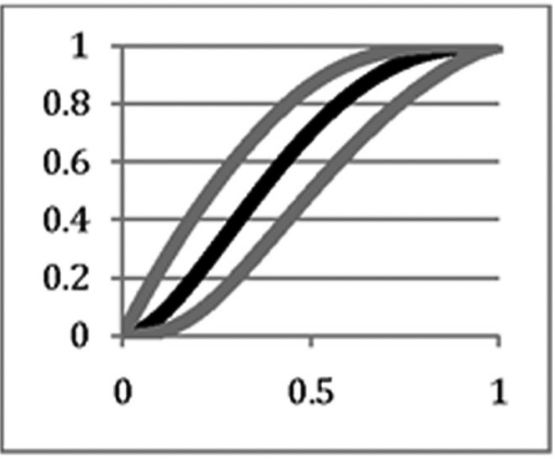

\section{MTT}

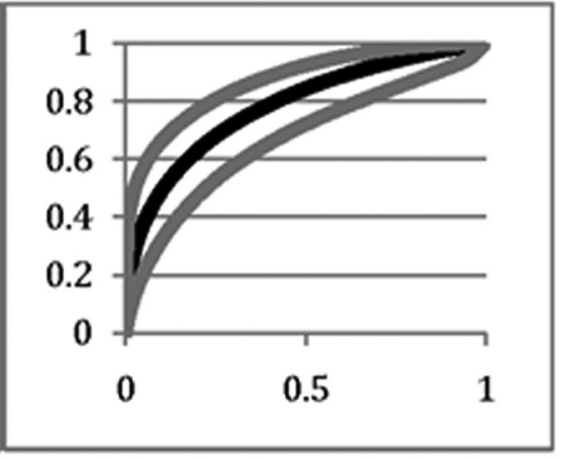

FIG 1. ROC curves for each CTP parameter for the outcomes of permanent neurologic deficits $(A)$, cerebral infarction $(B)$, and DCI (C). Gray curves indicate the upper and lower $95 \%$ confidence intervals.

\section{Primary Imaging Outcome: Cerebral Infarction}

Infarction occurred in $18 \%(17 / 96)$ of patients, and the remaining 82\% (79/96) had no infarction. Qualitative CTP deficits were seen in $88 \%(15 / 17)$ of patients who developed infarction and $41 \%(32 / 79)$ who did not $(P=.0004)$. The test characteristics (95\% CI) of qualitative CTP deficits for detecting patients who develop infarction were $88 \%(66 \%-97 \%)$ sensitivity, 59\% (48\%-70\%) specificity, and 32\% (20\%-46\%) positive and $96 \%(86 \%-99 \%)$ negative predictive values. Table 2 demonstrates the mean quantitative CTP parameters in these outcome groups. Figure $1 B$ displays the ROC curves for each CTP parameter for detecting patients who develop infarction. The diagnostic accuracy (SE) for each CTP parameter was 0.67 (0.07) for CBF, 0.53 (0.09) for CBV, and 0.72 (0.07) for MTT. A CBF threshold of $24 \mathrm{~mL} / 100 \mathrm{~g} / \mathrm{min}$ (73\% sensitivity, $50 \%$ specificity) and an MTT of 5.4 seconds (73\% sensitivity, 60\% specificity) were determined.

\section{Secondary Outcome: DCI}

DCI, defined as clinical deterioration, was determined in $50 \%$ (48/96) of patients, and the remaining 50\% (48/96) were without DCI. Qualitative CTP deficits occurred in $81 \%$ (39/48) of patients with DCI and $17 \%(8 / 48)$ of those without DCI $(P<.0001)$. The test characteristics $(95 \% \mathrm{CI})$ of qualitative CTP deficits for detecting DCI were $81 \%$ (68\%-90\%) sensitivity, 83\% (70\%-91\%) specificity, and $83 \%(70 \%-91 \%)$ positive and $82 \%(69 \%-90 \%)$ negative predictive values. Table 3 demonstrates the mean quantitative CTP parameters in these outcome groups. Figure $1 C$ displays the ROC curve for each CTP parameter for detecting DCI. The diagnostic accuracy (SE) for each CTP parameter was 0.81 
Table 3: Mean quantitative CTP parameters and SD for the secondary outcome measure of $\mathrm{DCl}^{\mathrm{a}}$

\begin{tabular}{|c|c|c|c|c|c|}
\hline & \multicolumn{2}{|c|}{$\begin{array}{c}\mathrm{DCl} \\
(n=48)\end{array}$} & \multicolumn{2}{|c|}{$\begin{array}{l}\text { No } \mathrm{DCl} \\
(n=48)\end{array}$} & \multirow[b]{2}{*}{$P$ Value } \\
\hline & Mean & SD & Mean & SD & \\
\hline CBF & 26.62 & 13.25 & 39.84 & 11.32 & $<.0001$ \\
\hline CBV & 1.83 & 0.67 & 2 & 0.5 & .1622 \\
\hline MTT & 7.2 & 3.5 & 4.7 & 1.4 & $<.0001$ \\
\hline
\end{tabular}

${ }^{\mathrm{a}} \mathrm{DCl}$ is defined as clinical deterioration.

(0.04) for CBF, 0.61 (0.06) for CBV, and 0.78 (0.05) for MTT. A CBF threshold of $30.5 \mathrm{~mL} / 100 \mathrm{~g} / \mathrm{min}$ (78\% sensitivity, $70 \%$ specificity) and an MTT of 5.0 seconds (72\% sensitivity, 70\% specificity) were determined.

The discharge status for the study population is shown in Online Table 1.

\section{Subgroup Analysis}

CTP was further evaluated in the DCI (symptomatic) and no DCI (asymptomatic) groups separately by using the primary outcome measures. In the DCI group, 54\% (26/48) of patients had permanent neurologic deficits and the remaining 46\% (22/48) did not. Qualitative CTP deficits were seen in 85\% (22/26) of patients who developed permanent neurologic deficits and in $77 \%(17 / 22)$ of those who did not $(P=.7131)$. Infarction occurred in 31\% (15/ $48)$ of patients, and the remaining $69 \%(33 / 48)$ were without it. Qualitative CTP deficits were seen in 93\% (14/15) of patients who developed infarction and in $76 \%$ (25/33) of those who did not $(P=.2389)$. Furthermore, there were no statistically significant differences in the mean quantitative CTP values between patients with and without permanent neurologic deficits or infarction in the DCI group (On-line Table 2).

In the no DCI group, $12 \%(6 / 48)$ of patients had permanent neurologic deficits and the remaining 88\% (42/48) did not. Qualitative CTP deficits were seen in 50\% (3/6) of patients who developed permanent neurologic deficits and in $12 \%(5 / 42)$ of those who did not $(P=.0497)$. Infarction occurred in $4 \%(2 / 48)$ of patients, and the remaining 96\% (46/48) were without. Qualitative CTP deficits were seen in 50\% (1/2) of patients who developed infarction and in 15\% (7/46) of those who did not $(P=$ .3085). Furthermore, there were no statistically significant differences in the mean quantitative $\mathrm{CBF}$ and CBV values between patients with and without permanent neurologic deficits or infarction in the no DCI group (On-line Table 3). However, there was a statistically significant difference in MTT for patients with permanent neurologic deficits $(P=.0196)$ and borderline significance for infarction $(P=.054)$.

\section{DISCUSSION}

DCI is a complex state described as clinical deterioration that may lead to cerebral infarction and permanent neurologic impairment. The diagnosis of DCI in aneurysmal SAH remains challenging due to the difficulty of adequately assessing critically ill and comatose patients. Therefore, imaging has been used as additional evidence to support a diagnosis of DCI, particularly in the management and treatment decision-making. Arterial narrowing on imaging studies, such as transcranial Doppler sonography, CTA, and DSA, has been used to detect vasospasm as proof of concept for the diagnosis of DCI. Current recommendations advise that vasospasm should be evaluated in conjunction with evidence of clinical deterioration. ${ }^{1}$ It is important to recognize that though severe vasospasm may decrease cerebral perfusion, it may not result in DCI.

In recent years, CTP has been added as complementary imaging to CTA and DSA to evaluate hemodynamic disturbances thought to occur in DCI. Perfusion deficits are described as focal regions of reduced CBF and/or prolonged MTT. However, global perfusion abnormalities have also been observed in patients with DCI. ${ }^{14}$ Few studies in the literature have evaluated CTP by using DCI defined as clinical deterioration. Dankbaar et $\mathrm{al}^{3}$ investigated the diagnostic value of CTP for DCI after clinical deterioration in 39 patients with SAH. Qualitative CTP was reported to have $84 \%$ (65\%-94\%) sensitivity, 79\% (52\%-92\%) specificity, and $88 \%$ $(69 \%-96 \%)$ positive and $73 \%(48 \%-89 \%)$ negative predictive values. ${ }^{3}$ In our larger cohort study, CTP deficits occurred more often in patients with DCI $(P<.0001)$ and had test characteristics similar to those previously reported. We found $81 \%(68 \%-90 \%)$ sensitivity, $83 \%(70 \%-91 \%)$ specificity, and $83 \%(70 \%-91 \%)$ positive and $82 \%(69 \%-90 \%)$ negative predictive values.

More recently, investigations have focused on using quantitative CTP and determining the best diagnostic threshold for determining DCI. Dankbaar et $\mathrm{al}^{4,15}$ reported significantly reduced $\mathrm{CBF}$ and prolonged MTT in patients with DCI. The diagnostic accuracy was calculated from the AUC as $71 \% \mathrm{CBF}, 57 \% \mathrm{CBV}$, and $76 \%$ MTT. $^{4}$ In this study, optimal diagnostic threshold values were reported as an MTT of 5.9 seconds (70\% sensitivity, 77\% specificity) and CBF of $36.3 \mathrm{~mL} / 100 \mathrm{~g} / \mathrm{min}$ (74\% sensitivity, $63 \%$ specificity). ${ }^{4}$ Again, our study revealed similar results with significantly reduced CBF and prolonged MTT in patients with DCI $(P<.0001)$. The diagnostic accuracy, also measured by the AUC, was highest for CBF (0.81) and MTT (0.78). Our threshold analysis revealed a CBF of $30.5 \mathrm{~mL} / 100 \mathrm{~g} / \mathrm{min}$ (78\% sensitivity, 70\% specificity) and an MTT of 5.0 seconds (72\% sensitivity, 70\% specificity).

There is limited literature for studies that have evaluated CTP by using outcome measures of DCI, as defined by the recent expert consensus. ${ }^{1}$ Pham et al ${ }^{16}$ reported that qualitative analysis of TTP maps demonstrated $93 \%$ sensitivity and $67 \%$ specificity for prediction of infarction. On quantitative analysis, the odds ratio (95\% CI) was $1.4(1.07-1.82)$ for 1 second of side-to-side delay in TTP. Furthermore, Lagares et $\mathrm{al}^{17}$ described acute perfusion changes after SAH and reported that an MTT of $>5.9$ seconds is associated with a 20 -fold risk of poor outcomes on the Glasgow Outcome Scale with a $90 \%$ positive predictive value. In our study, CTP deficits occurred more frequently in patients who developed poor outcomes of permanent neurologic deficits $(P<.0001)$ and infarction $(P=.0004)$, with high sensitivity and negative predictive values. Figure 2 demonstrates a qualitative CTP deficit in a patient who developed infarction. On the other hand, the high negative predictive values of CTP add valuable information in patients who may have lower probability of developing poor outcomes. Furthermore, quantitative analysis revealed again that CBF and MTT had the highest diagnostic accuracy for detecting patients who developed these primary outcome measures with similar threshold values determined for permanent neurologic 

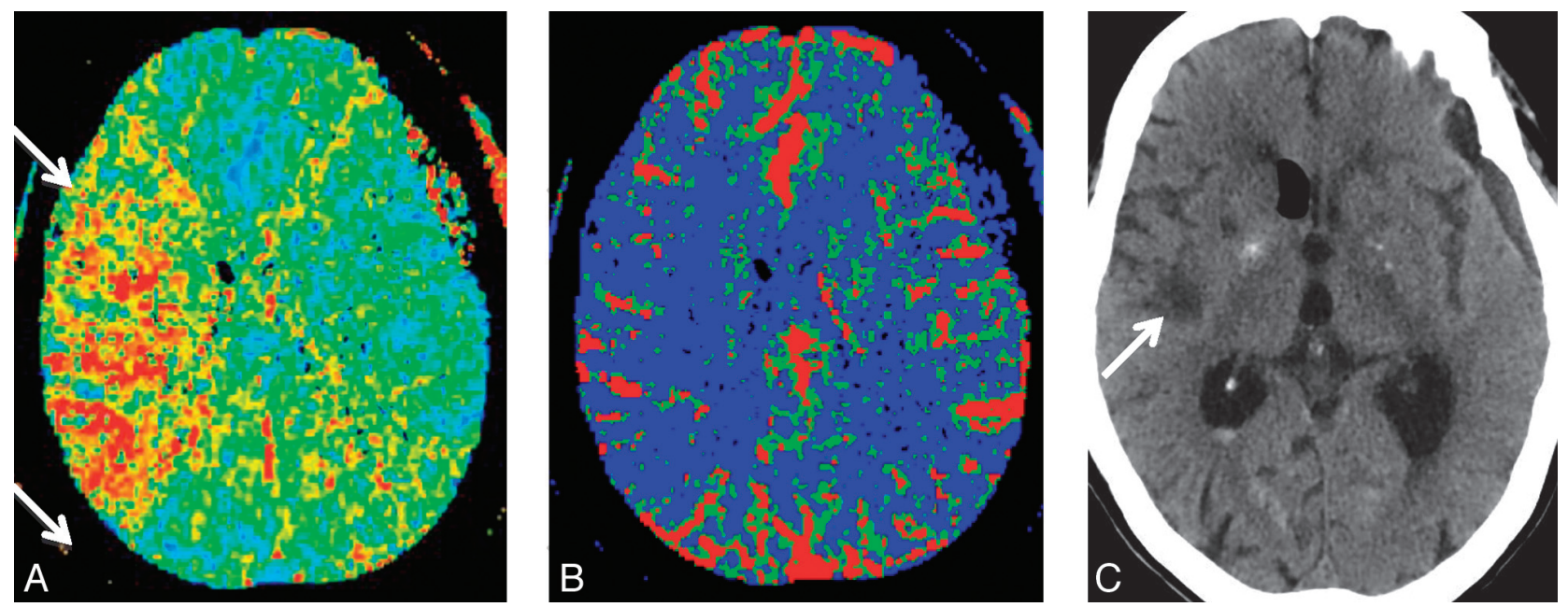

FIG 2. An 88-year-old woman status post surgical clipping of a left posterior communicating artery aneurysm. On day 7 , she developed left upper and lower extremity motor deficits. Initial noncontrast CT (not shown) revealed no evidence of acute infarction, new hemorrhage, or hydrocephalus to explain the new onset of symptoms. CTP demonstrates a large right-sided perfusion deficit with elevated MTT $(A)$ and reduced $C B F(B)$ in the right $M C A$ territory (arrows). Noncontrast $C T(C)$ performed on day 21 shows an infarction involving the right insular region (arrows) corresponding to the CTP deficit.

deficits and infarction. Specifically, the CBF threshold of 24-25 $\mathrm{mL} / 100 \mathrm{~g} / \mathrm{min}$ for permanent neurologic deficits and infarction is slightly lower than the CBF threshold for DCI, indicating the possibility of differentiating these outcome measures in future prospective studies.

In our subanalysis of patients with no DCI (asymptomatic), there was borderline significance of qualitative CTP deficits occurring more often in patients who developed permanent neurologic deficits $(P=.0497)$. Quantitative analysis demonstrated significantly prolonged MTT in patients who developed permanent neurologic deficits $(P=.0196)$ and borderline significance in MTT for infarction $(P=.054)$. However, in the patients with DCI (symptomatic), there were no significant differences in the qualitative or quantitative CTP for patients who had poor outcomes. This subanalysis is limited by the small sample sizes in the outcome groups. In addition, we found that the DCI group had significantly more patients discharged to rehabilitation facilities, whereas the no DCI group had more patients discharged to home. Although it was not statistically significant, there was also a trend toward increased mortality in the DCI group. These findings suggest that even with current treatment regimens, patients with DCI develop worse outcomes with increased morbidity and mortality.

We acknowledge several limitations in this study. We collected the outcomes data retrospectively. In addition, CTP provided limited brain coverage for the evaluation of perfusion deficits. However, emerging CT technology should continue to improve and provide broader coverage to which these findings may apply. Another limitation is that the region of CTP imaging was not coordinated with the location of the region referable to symptoms. Therefore, it is conceivable that this region may not have been imaged on CTP or was possibly averaged with a larger region of normal perfusion, resulting in increased false-negatives and lower accuracy in our study. We did not use clinical information to select the region of imaging, to reduce work-up and observer bias by not having knowledge of the clinical examination before
CTP scanning and interpretation. Last, there is an inherent limitation in using quantitative CTP findings for clinical decisionmaking. Careful interpretation of absolute threshold values is recommended because these are only valid for the constellation of the type of hardware equipment and software programs used in a specific study. Thereby, standardization and validation of CTP methodology and postprocessing techniques are necessary for its widespread implementation in patients with SAH.

\section{CONCLUSIONS}

CTP may provide hemodynamic evidence to support the diagnosis of DCI and prognostic information of poor outcomes in patients with aneurysmal SAH. CTP deficits occurred more often in patients who developed DCI, permanent neurologic deficits, and infarction. Quantitative CTP demonstrated significantly reduced CBF and prolonged MTT in patients who developed DCI and had poor outcomes. Even with current treatment regimens, patients with DCI are more likely to have worse outcomes, with increased morbidity and mortality. These initial findings support the need for prospective clinical research focused on developing CTP as a prognostic tool for DCI and poor outcomes, which may provide valuable information in the management and treatment of aneurysmal SAH.

Disclosures: Alan Segal—UNRELATED: Consultancy: Maquet Cardiovascular, Expert Testimony: opinion in nonrelated medical malpractice litigation. Robert D. Zimmer man—RELATED: Grant: NIH.* Alvin Mushlin—RELATED: Consulting Fee or Honorar ium: Association of University Radiologists, Comments: serves on the advisory board for the academic fellowship for radiologist training in technology assessment and health services research; the GE Academic Radiology Research Fellowship, $\$ 3000.00$, UNRELATED: Consultancy: travel to 1 annual meeting to mentor the GERRAF fellows. *Money paid to the institution.

\section{REFERENCES}

1. Vergouwen MD, Vermeulen M, van Gijn J, et al. Definition of delayed cerebral ischemia after aneurysmal subarachnoid hemorrhage as an outcome event in clinical trials and observational studies: proposal of a multidisciplinary research group. Stroke 2010;41:2391-95

AJNR Am J Neuroradiol 34:292-98 Feb 2013 www.ajnr.org 
2. Dankbaar JW, Rijsdijk M, van der Schaaf IC, et al. Relationship between vasospasm, cerebral perfusion, and delayed cerebral ischemia after aneurysmal subarachnoid hemorrhage. Neuroradiology 2009;51:813-19

3. Dankbaar JW, de Rooij NK, Velthuis BK, et al. Diagnosing delayed cerebral ischemia with different CT modalities in patients with subarachnoid hemorrhage with clinical deterioration. Stroke 2009;40: 3493-98

4. Dankbaar JW, de Rooij NK, Rijsdijk M, et al. Diagnostic threshold values of cerebral perfusion measured with computed tomography for delayed cerebral ischemia after aneurysmal subarachnoid hemorrhage. Stroke 2010;41:1927-32

5. Aralasmak A, Akyuz M, Ozkaynak C, et al. CT angiography and perfusion imaging in patients with subarachnoid hemorrhage: correlation of vasospasm to perfusion abnormality. Neuroradiology 2009;51:85-93

6. Sanelli PC, Ugorec I, Johnson CE, et al. Using quantitative CT perfusion for evaluation of delayed cerebral ischemia following aneurysmal subarachnoid hemorrhage. AJNR Am J Neuroradiol 2011;32: 2047-53

7. Wintermark M, Ko NU, Smith WS, et al. Vasospasm after subarachnoid hemorrhage: utility of perfusion CT and CT angiography on diagnosis and management. AJNR Am J Neuroradiol 2006;27:26-34

8. Frontera JA, Fernandez A, Schmidt JM, et al. Defining vasospasm after subarachnoid hemorrhage: what is the most clinically relevant definition? Stroke 2009;40:1963-68

9. Powsner RA, O’Tuama LA, Jabre A, et al. SPECT imaging in cerebral vasospasm following subarachnoid hemorrhage. J $\mathrm{Nucl} \mathrm{Med}$ 1998;39:765-69
10. Wintermark M, Maeder P, Thiran JP, et al. Quantitative assessment of regional blood flows by perfusion CT studies at low injection rates: a critical review of the underlying theoretical models. Eur Radiol 2001;11:1220-30

11. Sanelli PC, Lev MH, Eastwood JD, et al. The effect of varying userselected input parameters on quantitative values in CT perfusion maps. Acad Radiol 2004;11:1085-92

12. Wintermark M, Lau BC, Chien J, et al. The anterior cerebral artery is an appropriate arterial input function for perfusion-CT processing in patients with acute stroke. Neuroradiology 2008;50:227-36

13. Murphy BD, Fox AJ, Lee DH, et al. Identification of penumbra and infarct in acute ischemic stroke using computed tomography perfusion-derived blood flow and blood volume measurements. Stroke 2006;37:1771-77

14. Rijsdijk M, van der Schaaf IC, Velthuis BK, et al. Global and focal cerebral perfusion after aneurysmal subarachnoid hemorrhage in relation with delayed cerebral ischemia. Neuroradiology 2008;50: 813-20

15. Dankbaar JW, de Rooij NK, Smit EJ, et al. Changes in cerebral perfusion around the time of delayed cerebral ischemia in subarachnoid hemorrhage patients. Cerebrovasc Dis 2011;32:133-40

16. Pham M, Johnson A, Bartsch AJ, et al. CT perfusion predicts secondary cerebral infarction after aneurysmal subarachnoid hemorrhage. Neurology 2007;69:762-65

17. Lagares A, Cicuendez M, Ramos A, et al. Acute perfusion changes after spontaneous SAH: a perfusion CT study. Acta Neurochir (Wien) 2012;154:405-12 